\title{
GDNF Protection against 6-OHDA-Induced Reductions in Potassium-Evoked Overflow of Striatal Dopamine
}

\author{
Wayne A. Cass and Michael W. Manning \\ Department of Anatomy and Neurobiology, University of Kentucky College of Medicine, Lexington, Kentucky 40536-0298
}

Glial cell line-derived neurotrophic factor (GDNF), when administered before 6-hydroxydopamine (6-OHDA), has been shown to prevent the reduction in nigral dopamine (DA) levels and tyrosine hydroxylase-positive neurons normally observed after 6-OHDA lesions. The present study examined the ability of GDNF to prevent 6-OHDA-induced reductions in striatal DA release and reductions in striatal and nigral DA levels. GDNF (10 $\mu \mathrm{g}$ ), or vehicle, was injected into the right nigra of anesthetized male Fischer-344 rats and was followed $6 \mathrm{hr}$ later by intranigral 6-OHDA or saline. Three to four weeks later the animals were anesthetized with urethane and prepared for in vivo electrochemistry. Potassium-evoked overflow of DA was dramatically decreased in the right striatum of the vehicle + 6-OHDAtreated animals. GDNF appeared to prevent the reduction in evoked overflow of DA in the right striatum of the 6-OHDA- treated animals. However, in comparison with that in animals that received GDNF + saline, the overflow of DA was significantly reduced in the GDNF + 6-OHDA animals. Similarly, although nigral levels of DA were above normal in the GDNF + 6-OHDA-treated animals, they were below DA levels found in GDNF + saline-treated rats. Striatal DA levels were partially protected by GDNF. In animals examined 10-12 weeks after the GDNF and 6-OHDA treatments, the apparent protective ability of GDNF on the evoked overflow of DA in the striatum was diminished. Thus, although intranigral GDNF can prevent 6-OHDA-induced reductions in nigral DA levels, long-term protection of the evoked overflow of DA in the striatum is minimal.

Key words: GDNF; 6-hydroxydopamine; striatum; dopamine; neurotrophic factor; in vivo electrochemistry; neurotoxicity
Parkinson's disease is characterized by the progressive degeneration of nigrostriatal dopamine (DA) neurons. Drugs or other compounds that can slow down this degeneration or partially restore dopaminergic functioning would therefore be of potential therapeutic benefit. One such possible compound is glial cell line-derived neurotrophic factor (GDNF). In vitro, GDNF promotes the survival and differentiation of embryonic, mesencephalic DA neurons (Lin et al., 1993; Hou et al., 1996). In normal young and aged animals, in vivo GDNF can augment nigral DA levels and tyrosine hydroxylase (TH)-positive fiber density (Gash et al., 1995; Hudson et al., 1995; Martin et al., 1996) and can increase evoked overflow of DA in the striatum (Gash et al., 1995; Hebert et al., 1996; Hebert and Gerhardt, 1997). When given after administration of the neurotoxins 6-hydroxydopamine (6OHDA) (Hoffer et al., 1994; Bowenkamp et al., 1995; Lapchak et al., 1997; Rosenblad et al., 1998) or 1-methyl-4-phenyl-1,2,3,6tetrahydropyridine (MPTP) (Tomac et al., 1995; Gash et al., 1996; Miyoshi et al., 1997; Zhang et al., 1997), GDNF can partially ameliorate the behavioral and neurochemical deficits normally observed in these animals. All of these studies emphasize the potential therapeutic application of GDNF for treating Parkinson's disease.

Several studies have examined the neuroprotective potential of GDNF against lesions of the nigrostriatal DA system. For instance, GDNF has been shown to provide varying degrees of

\footnotetext{
Received July 17, 1998; revised Oct. 27, 1998; accepted Nov. 23, 1998.

This work was supported in part by the American Parkinson Disease Association. We thank Synergen (Boulder, CO) and Amgen (Thousand Oaks, CA) for the generous gift of GDNF.

Correspondence should be addressed to Dr. Wayne A. Cass, Department of Anatomy and Neurobiology, MN 224 Chandler Medical Center, University of Kentucky, Lexington, KY 40536-0298.

Copyright (C) 1999 Society for Neuroscience $\quad 0270-6474 / 99 / 191416-08 \$ 05.00 / 0$
}

protection to DA neurons against MPTP toxicity (Tomac et al., 1995; Kojima et al., 1997), 6-OHDA toxicity (Kearns and Gash, 1995; Sauer et al., 1995; Winkler et al., 1996; Choi-Lundberg et al., 1997; Sullivan et al., 1998), and methamphetamine toxicity (Cass, 1996). In addition, GDNF can also reduce degeneration of mesencephalic DA neurons after transection of the medial forebrain bundle (Beck et al., 1995). However, the extent of protection by GDNF varies markedly in different studies, pointing out that several factors are important for determining the success of GDNF in protecting DA neurons. These factors include type and extent of the lesion as well as dose, site of injection, and the time course for delivery of GDNF.

One recent study has shown that a single intranigral injection of GDNF, given $6 \mathrm{hr}$ before intranigral 6-OHDA, can prevent completely the 6-OHDA-induced loss of DA neurons and DA content in the substantia nigra (Kearns et al., 1997). However, whether or not the functional dynamics of DA release and uptake in the striatum of these animals is also preserved is unclear. The purpose of the present study was to evaluate further the protective effects of GDNF, given $6 \mathrm{hr}$ before a 6-OHDA lesion, on the nigrostriatal DA system. In vivo electrochemistry was used to measure potassium-evoked overflow of DA in the striatum at both 3-4 and 10-12 weeks after unilateral GDNF and 6-OHDA administration into the nigra. In addition, tissue levels of DA in the striatum and substantia nigra were determined at the conclusion of each experiment to evaluate the extent of the lesion produced by 6-OHDA and the ability of GDNF to preserve DA levels.

\section{MATERIALS AND METHODS}

Animals. Forty-eight male Fischer-344 rats (Harlan Sprague Dawley, Indianapolis, IN) weighing 230-330 gm were used for these experiments. They were housed in groups of two under a $12 \mathrm{hr}$ light/dark cycle with 
food and water available ad libitum. All animal-use procedures were in strict accordance with the National Institutes of Health Guide for the Care and Use of Laboratory Animals and were approved by the Animal Care and Use Committee at the University of Kentucky.

GDNF and 6-OHDA administration. Rats were anesthetized with sodium pentobarbital $(50 \mathrm{mg} / \mathrm{kg}$, i.p.) and placed into a stereotaxic frame. All surgery was performed using aseptic conditions. The skull was exposed, and a small hole was drilled in the skull over the right substantia nigra. Human recombinant GDNF (10 $\mu \mathrm{g}$ in $2 \mu \mathrm{l}$ of vehicle solution; Synergen, Boulder, CO, and Amgen, Thousand Oaks, CA) or $2 \mu \mathrm{l}$ of vehicle $(10 \mathrm{~mm}$ citrate buffer with $150 \mathrm{~mm} \mathrm{NaCl}, \mathrm{pH} 5)$ was injected into the dorsal region of the right substantia nigra $(5.4 \mathrm{~mm}$ posterior to bregma, $2.2 \mathrm{~mm}$ lateral from the midline, and $7.3 \mathrm{~mm}$ below the surface of the cortex) using a Hamilton or an SGE syringe with a 26 gauge needle. The rate of injection was $0.2 \mu \mathrm{l} / \mathrm{min}$. The needle was left in place for an additional 5 min after the injection and then slowly withdrawn. The scalp was closed with wound clips. Six hours later the animal was readministered sodium pentobarbital $(25 \mathrm{mg} / \mathrm{kg}$, i.p., or more if needed) and placed back into the stereotaxic frame. 6-OHDA hydrobromide ( 8 $\mu \mathrm{g}$ in $2 \mu \mathrm{l}$ of vehicle; Sigma, St Louis, MO) or $2 \mu \mathrm{l}$ of saline vehicle $(0.02 \%$ ascorbic acid in $0.9 \%$ saline) was injected using the same coordinates and protocol described above. After the injection, gelfoam was placed in the burr hole, the incision was sutured closed, and the animals were returned to their home cages for recovery.

In vivo electrochemistry. Three to four or $10-12$ weeks after the intranigral injections, the animals were anesthetized with urethane $(1.25-1.5 \mathrm{gm} / \mathrm{kg}$, i.p.), placed into a stereotaxic frame, and prepared for in vivo electrochemical recordings as described previously (Cass, 1996, 1997).

Nafion-coated single-carbon fiber electrodes were constructed and calibrated in vitro (Cass, 1996, 1997). The electrodes were attached to single-barrel micropipettes (tip diameter, $10-20 \mu \mathrm{m}$ ) with dental wax so that the tips of the pipettes and the electrodes were separated by $260-300$ $\mu \mathrm{m}$. The micropipettes were filled with a solution containing $70 \mathrm{~mm} \mathrm{KCl,}$ $79 \mathrm{~mm} \mathrm{NaCl}$, and $2.5 \mathrm{~mm} \mathrm{CaCl}_{2}$, pH 7.4.

High-speed chronocoulometric electrochemical measurements were made continuously at $5 \mathrm{~Hz}$ and averaged to $1 \mathrm{~Hz}$ using an IVEC-10 system (Medical Systems Corporation, Greenvale, NY). The applied oxidation potential was $+0.55 \mathrm{~V}$ for $100 \mathrm{msec}$ (vs $\mathrm{Ag} / \mathrm{AgCl}$ reference electrodes), and the resting potential was $0.0 \mathrm{~V}$ for $100 \mathrm{msec}$. The oxidation and reduction currents were digitally integrated during the last $80 \mathrm{msec}$ of each $100 \mathrm{msec}$ pulse. Electrode assemblies were initially positioned in the dorsal striatum (1.2-1.6 $\mathrm{mm}$ anterior to bregma, 2.2-2.3 $\mathrm{mm}$ lateral from the midline, and $3.5 \mathrm{~mm}$ below the surface of the brain). The baseline electrochemical signal was allowed to stabilize (5-10 min), and then $125-150 \mathrm{nl}$ of potassium solution $\left(70 \mathrm{mM} \mathrm{K}^{+}\right)$was applied by pressure ejection (Picospritzer II; General Valve, Fairfield, NJ) during the recordings to evoke the release of DA (see Fig. $1 B$ ). The volume of fluid injected was monitored by determining the amount of fluid displaced from the micropipette using a dissection microscope fitted with a reticule eyepiece and was based on previous calculations that there are $\sim 250 \mathrm{nl}$ of solution in a $1 \mathrm{~mm}$ segment of the micropipette (Friedemann and Gerhardt, 1992). After the signal had returned to baseline, the electrode and micropipette assembly was lowered by $0.5 \mathrm{~mm}$. The new baseline was allowed to stabilize, and then $125-150 \mathrm{nl}$ of potassium solution was applied again. The potassium applications were repeated at $0.5 \mathrm{~mm}$ steps to map the striatum and nucleus accumbens (see Fig. $1 A$ ). A single recording pass was made in each hemisphere of each animal. The order of the passes was alternated between animals in each group. There was no difference in the amount of potassium solution applied between the right and left sides of the brain or between the different treatment groups.

Tissue collection and HPLC analysis. At the end of the experiments, the animals were killed by decapitation while still anesthetized with urethane. The brains were rapidly removed and chilled in ice-cold saline. A coronal slice of brain $\sim 2 \mathrm{~mm}$ thick at the level of the recording tracts was made with the aid of a chilled brain mold (Rodent Brain Matrix; ASI Instruments, Warren, MI). The location of each recording electrode was confirmed by noting blood left in the recording track. The striatum was dissected from each half of the slice as a single piece. The substantia nigra was similarly dissected from both sides of a 2 -mm-thick coronal slice through the midbrain. The tissue pieces were placed in preweighed vials, weighed, and frozen on dry ice. Samples were stored at $-80^{\circ} \mathrm{C}$ until assayed by HPLC. Tissue levels of DA were determined using HPLC with electrochemical detection as described previously (Cass, 1996,
1997). Retention times of standards were used to identify peaks, and peak heights were used to calculate the recovery of internal standard (dihydroxybenzylamine) and the amount of DA. Results were expressed as micrograms per gram wet weight of tissue.

Data analysis. The electrodes used in this study, although relatively insensitive to ascorbic acid because of the Nafion coating (Gerhardt et al., 1984), can still detect serotonin (5-HT) if the levels are high enough. To confirm that the responses detected were attributable primarily to DA, we recorded both the reduction and oxidation currents and calculated the ratio of the reduction current to the oxidation current for each $\mathrm{K}^{+}$-induced response. The electrodes used in this study exhibit reduction/oxidation current ratios of 0.4 or greater for DA and ratios of 0.1-0.2 for 5-HT (Luthman et al., 1993; Cass, 1997). Ascorbic acid, if detected, is not reduced at the potentials used and therefore gives a reduction/oxidation current ratio of 0.0 . All of the responses included in the Results had reduction/oxidation ratios of at least 0.4 , indicating that DA was the predominant compound detected by the electrodes after potassium application.

For statistical analysis of the electrochemistry data two parameters were analyzed, (1) the maximum amplitude of the signals resulting from the application of potassium and (2) the clearance rate of the signals [the slope of the linear, declining portion of the signal between the $T_{20}$ and $T_{60}$ time points (see Fig. $1 \mathrm{~B}$ ), with the $T_{20}$ and $T_{60}$ points representing the time points at which the signal has declined by 20 and $60 \%$ of the maximum amplitude]. The clearance rate is an estimate of the maximal slope of the declining phase of the signal and is related to those processes responsible for removing DA from the extracellular space. This includes predominantly DA reuptake, but diff usion (and possibly metabolism) will play a greater role when there are fewer functional DA transporters in the region of the recording electrode (Wightman and Zimmerman, 1990; Cass et al., 1993; Suaud-Chagny et al., 1995; Garris et al., 1997). Signal amplitude, clearance rate, and tissue monoamine concentration were analyzed using $t$ tests or ANOVAs, followed by Newman-Keuls post hoc comparisons as indicated in the Results.

\section{RESULTS}

\section{Three to four weeks after the lesion}

Locally applied potassium produced signals with reduction/oxidation current ratios characteristic for DA at all recording depths in the striatum and nucleus accumbens of all animals. Representative responses from the right striatum of GDNF + 6-OHDAand of vehicle +6 -OHDA-treated rats are shown in Figure $1 B$. On the basis of observations of the placement of the electrode tracts in the present study and previous experience with recording from the striatum and nucleus accumbens (Cass et al., 1992, 1993; Cass and Gerhardt 1994, 1995), recordings at a depth of 3.5-6.0 $\mathrm{mm}$ below the surface of the cortex are contained within the striatum (Fig. 1A). Recordings at a depth of $6.5 \mathrm{~mm}$ are approximately at the junction of the striatum and the nucleus accumbens, whereas recordings at depths of 7.0 and $7.5 \mathrm{~mm}$ are within the nucleus accumbens.

Signal amplitudes from animals treated with vehicle or GDNF followed $6 \mathrm{hr}$ later by saline injections are shown in Figure 2. In the vehicle + saline-treated animals, there was no difference at any individual recording depth between the signals recorded from the treated side of the brain and the contralateral side. However, within each side, signal amplitude did drop off at the deeper recording sites, particularly in the region of the nucleus accumbens ( $p<0.05$, one-way repeated measures ANOVA). This is similar to what has been reported previously (Cass, 1996, 1997; Hebert et al., 1996) and is likely caused by the decreased density of dopaminergic innervation to the nucleus accumbens and adjacent striatum. In the GDNF + saline-treated animals, there was a tendency for signal amplitude to be augmented on the GDNFtreated side of the brain compared with the contralateral side (Fig. 2). However, this increase did not reach statistical significance. As with the vehicle + saline-treated animals, there was a significant difference in signal amplitude over depth on both the 


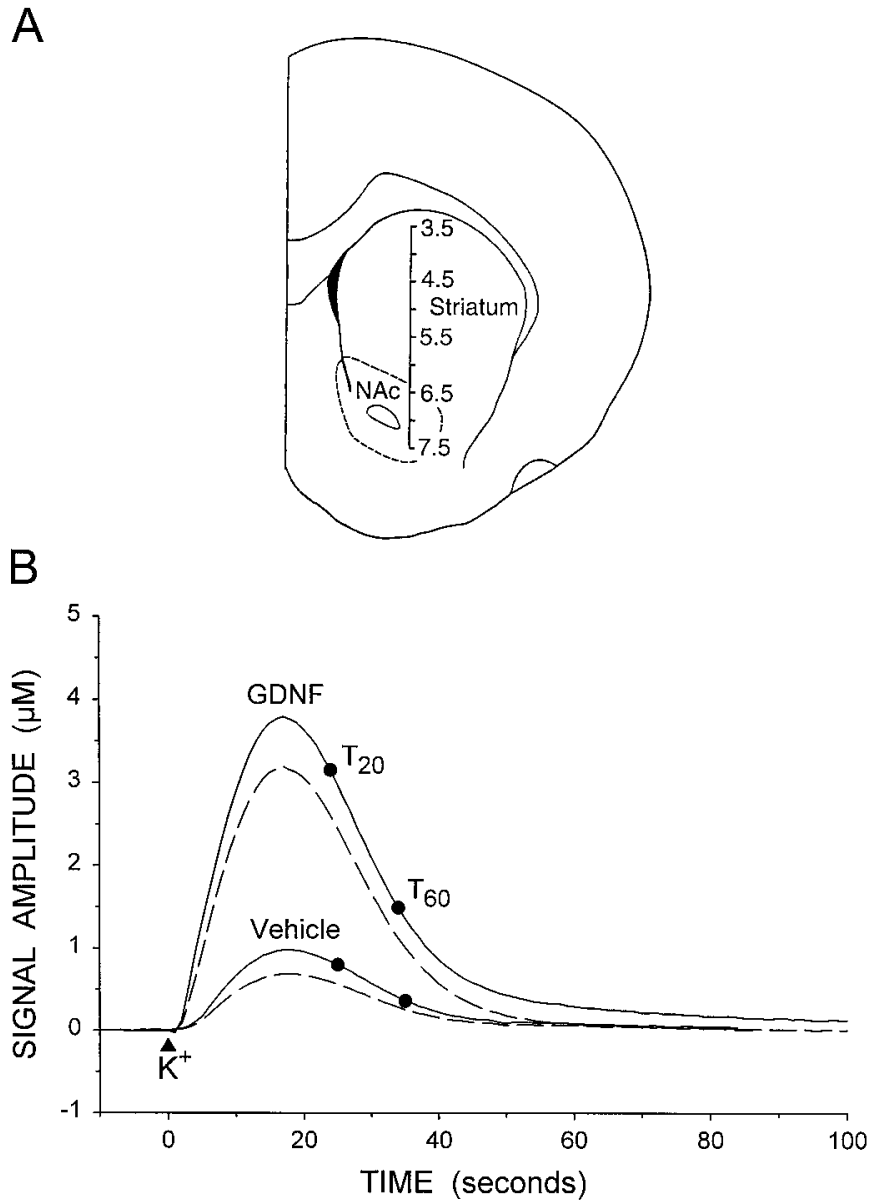

Figure 1. A, Illustration of the locations of the recording sites for the in vivo electrochemical recordings. Data were collected at $0.5 \mathrm{~mm}$ steps throughout the dorsoventral extent of the striatum and nucleus accumbens ( $N A c)$ (3.5-7.5 mm below the surface of the brain) on both sides of the brain. This diagram represents a coronal section of the right hemisphere of the forebrain $\sim 1.2 \mathrm{~mm}$ anterior to bregma. $B$, Representative signals from the dorsal striatum of a GDNF + 6-OHDA-treated rat $(G D N F)$ and a vehicle +6 -OHDA-treated rat (Vehicle) showing the potassium-evoked overflow of DA. Potassium $\left(150 \mathrm{nl} ; 70 \mathrm{mM} \mathrm{K}^{+}\right)$was applied at the arrowhead at time 0 in both cases. The solid lines are the oxidation current responses, and the dashed lines below each solid line are the corresponding reduction current responses. The reduction/oxidation current ratios ( 0.83 and 0.70 for the GDNF and Vehicle responses, respectively) indicate that the predominate electroactive species detected was DA. The $T_{20}$ and $T_{60}$ time points used for calculating clearance rate are indicated on the oxidation curve of both recordings and are labeled on the curve from the GDNF + 6-OHDA-treated rat.

ipsilateral and contralateral sides of the GDNF + saline-treated rats (one-way repeated measures ANOVA).

In the animals treated with vehicle followed by 6-OHDA, there was a dramatic decrease in the amplitude of the signals on the lesioned side (Fig. 3). These reductions were significant in the dorsal striatum but not in the nucleus accumbens. In the GDNF + 6-OHDA-treated animals, the amplitude of the potassiumevoked signals tended to be decreased in the dorsal striatum on the treated side of the brain, but there was no main effect of treatment in the overall ANOVA (Fig. 3). However, if the signal amplitudes from the treated side of the brain of the GDNF + 6-OHDA group are compared with that from the treated side of the GDNF + Saline group (Fig. 2), there is a significant effect of treatment (two-way ANOVA with depth below the brain surface
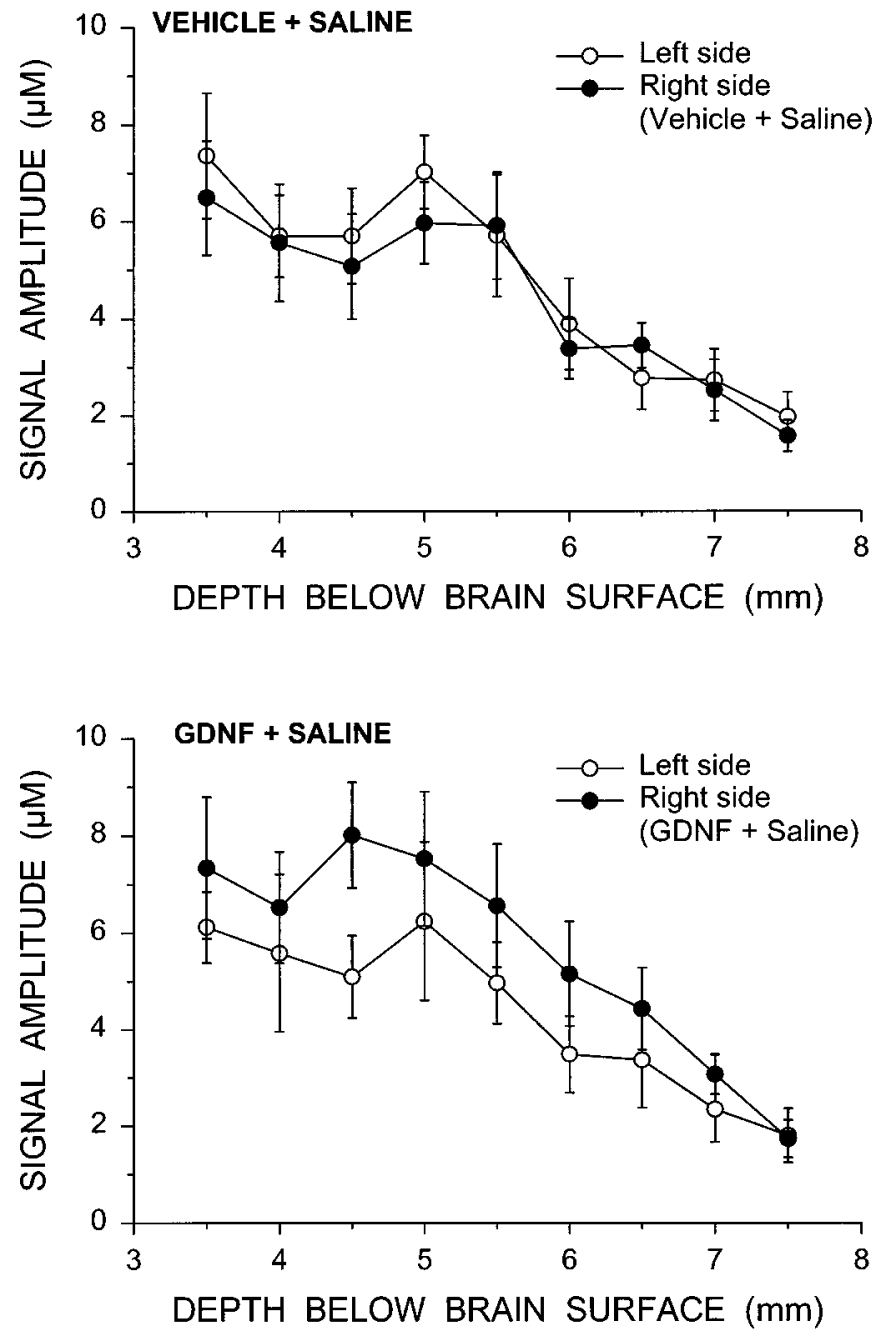

Figure 2. Summary of potassium-evoked DA signal amplitude throughout the striatum and nucleus accumbens of animals treated with vehicle (top) or GDNF (bottom) followed $6 \mathrm{hr}$ later by saline. All injections were made into the right substantia nigra. In vivo electrochemical recordings were made 3-4 weeks after the intranigral injections. The data shown are mean $( \pm$ SEM) values for eight animals per group. The data were analyzed using two-factor ANOVA with side of the brain and depth of the recording as within factors. $F$ scores for the vehicle + saline group are as follows: side $F=0.13, p>0.05$; depth $F=13.31, p<0.001$; and interaction $F=0.36, p>0.05$. GDNF + saline group $F$ scores are as follows: side $F=2.16, p>0.05$; depth $F=12.02, p<0.001$; and interaction $F=0.63, p>0.05$.

as a within factor; treatment $F=6.45, p=0.024$; depth $F=8.78$, $p<0.001$; and interaction $F=2.35, p=0.023$ ). Similarly, there is also a significant effect of treatment between the treated sides of the GDNF + 6-OHDA group and the vehicle + 6-OHDA group (treatment $F=11.17, p=0.005$; depth $F=1.51, p=0.16$; and interaction $F=2.01, p=0.051)$.

The ANOVA results indicated that the 6-OHDA treatment in the vehicle +6 -OHDA animals decreased DA release in the dorsal striatum but not in the nucleus accumbens. Because only the striatum was effected, data from recordings at depths from 3.5 to $6.0 \mathrm{~mm}$ below the surface of the brain were averaged together for each animal to obtain an overall value for the striatum. When this was done, both signal amplitude and clearance rate were decreased on the treated side of the vehicle +6-OHDA group by $83 \%$ (Fig. 4). On the right side of the GDNF + 6-OHDA-treated 

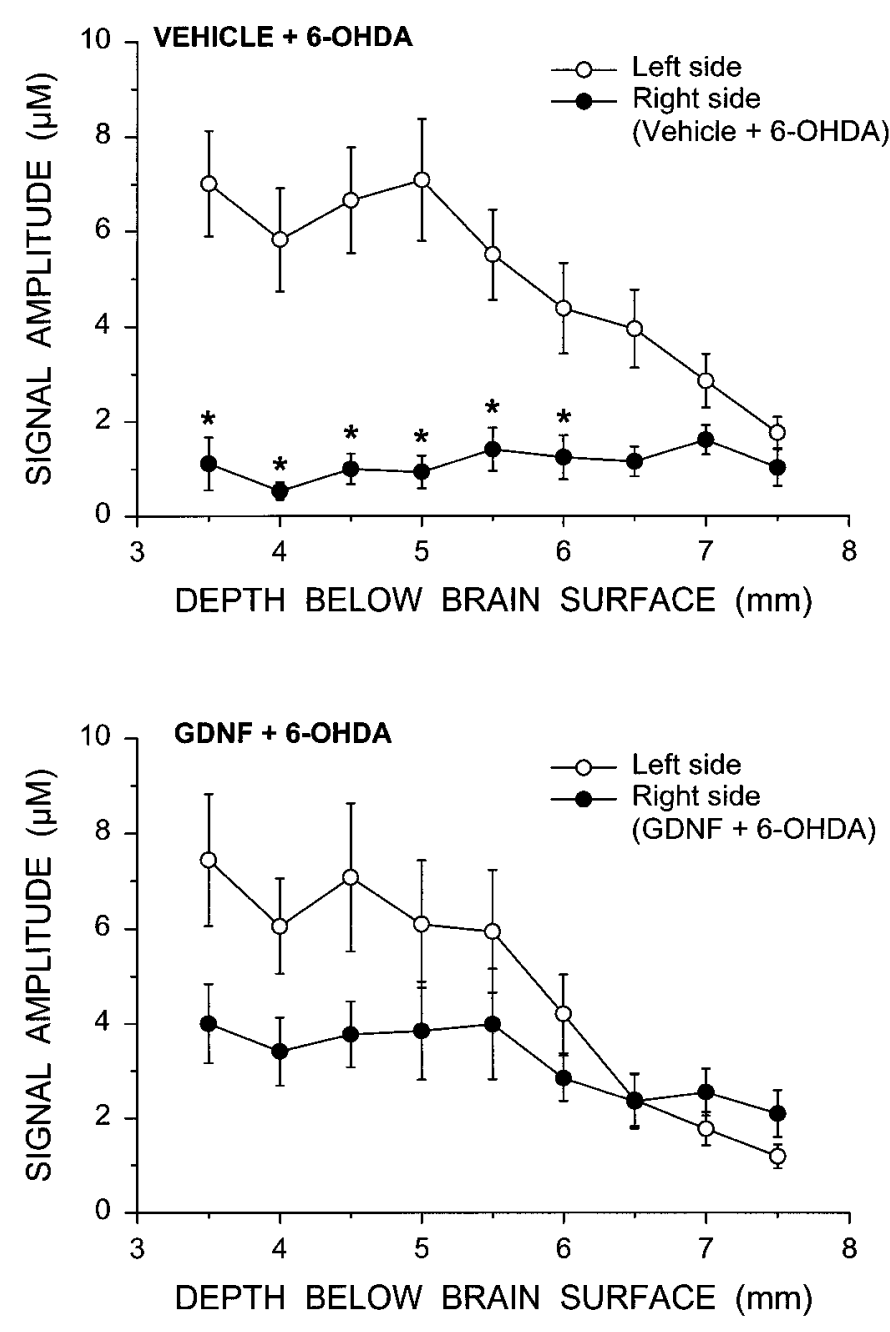

Figure 3. Summary of potassium-evoked DA signal amplitude throughout the striatum and nucleus accumbens of animals treated with vehicle (top) or GDNF (bottom) followed $6 \mathrm{hr}$ later by 6-OHDA. All injections were made into the right substantia nigra. In vivo electrochemical recordings were made 3-4 weeks after the intranigral injections. The data shown are mean $( \pm$ SEM) values for eight animals per group. The data were analyzed using two-factor ANOVA with side of the brain and depth of the recording as within factors. $F$ scores for the vehicle $+6-O H D A$ group are as follows: side $F=56.65, p<0.001$; depth $F=7.07, p<0.001$; and interaction $F=7.83, p<0.001 . G D N F+6-O H D A$ group $F$ scores are as follows: side $F=2.94, p>0.05$; depth $F=12.03, p<0.001$; and interaction $F=3.13, p<0.01$. ${ }^{*} p<0.05$ versus the left side at the same depth (Newman-Keuls post hoc comparisons).

animals, signal amplitude was decreased by $41 \%$, and clearance rate was decreased by $51 \%$ (Fig. 4). Both signal amplitude and clearance rate were significantly greater on the right side of the GDNF + 6-OHDA-treated animals than on the right side of the vehicle +6 -OHDA-treated animals.

DA levels in the striatum and substantia nigra are shown in Figure 5. In the striatum there was a $19 \%$ decrease in DA levels on the treated side of the GDNF + saline animals. In the vehicle +6 -OHDA animals, there was an $81 \%$ decrease in DA levels on the treated side, and in the GDNF + 6-OHDA animals, there was a $48 \%$ decrease on the treated side. In the substantia nigra of the GDNF + saline-treated animals, there was a 92\% increase in DA content on the right side. In the vehicle + 6-OHDA group, nigral DA levels were reduced by $76 \%$ on the lesioned side, whereas on the treated side of the GDNF + 6-OHDA-treated animals, there
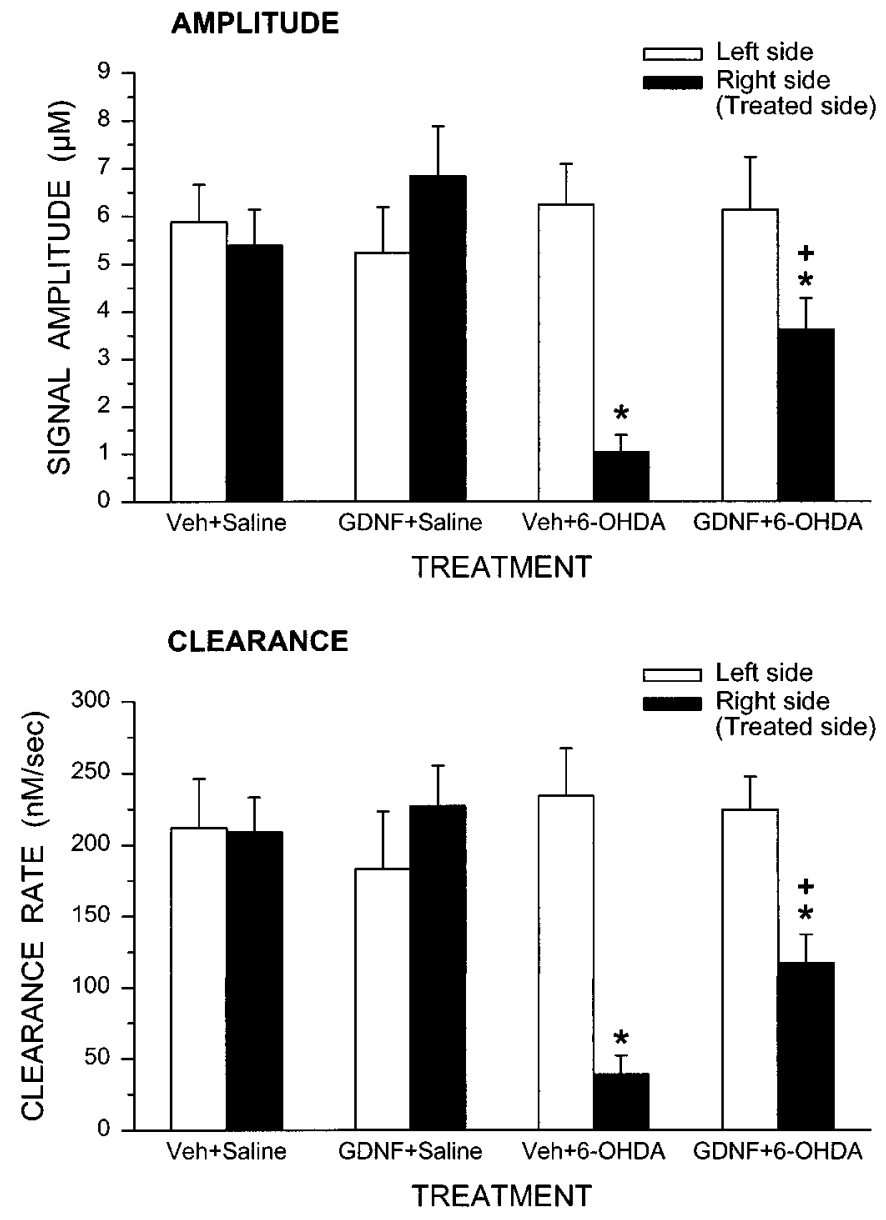

Figure 4. Signal amplitudes (top) and clearance rates (bottom) from the striatum of rats treated with $G D N F$ or vehicle $(V e h)$ followed $6 \mathrm{hr}$ later by 6-OHDA or saline. All injections were made into the right substantia nigra. In vivo electrochemical recordings were made 3-4 weeks after the injections. The data from each recording depth in the striatum (3.5-6.0 $\mathrm{mm}$ below the surface of the cortex) of each animal were averaged together. The values shown are mean $( \pm$ SEM) for eight animals per group. ${ }^{*} p<0.05$ versus the left side of the same group; $+p<0.05$ versus the right side of the vehicle $+6-O H D A$ group (two-factor ANOVA with side of the brain as a within factor; Newman-Keuls post hoc comparisons).

was a trend for an increase in nigral DA levels. In both the striatum and the nigra, DA levels on the right side of the GDNF

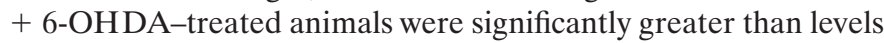
on the right side of the vehicle $+6-O H D A-t r e a t e d$ animals.

\section{Ten to twelve weeks after the lesion}

The results at 3-4 weeks after treatment suggest that the GDNF is partially protecting striatal DA terminals against the effects of 6-OHDA and possibly providing complete protection to DA cell bodies in the substantia nigra. However, another possibility is that the GDNF is just upregulating the remaining nigrostriatal neurons rather than protecting against the effects of 6-OHDA. To examine this possibility, a second set of experiments was performed in which rats were treated with GDNF + saline or with GDNF + 6-OHDA and 10-12 weeks were allowed to elapse before the in vivo electrochemistry experiments and the determination of striatal and nigral DA content.

In animals treated with GDNF + saline 10-12 weeks earlier, there was no difference in the potassium-evoked signal amplitude 

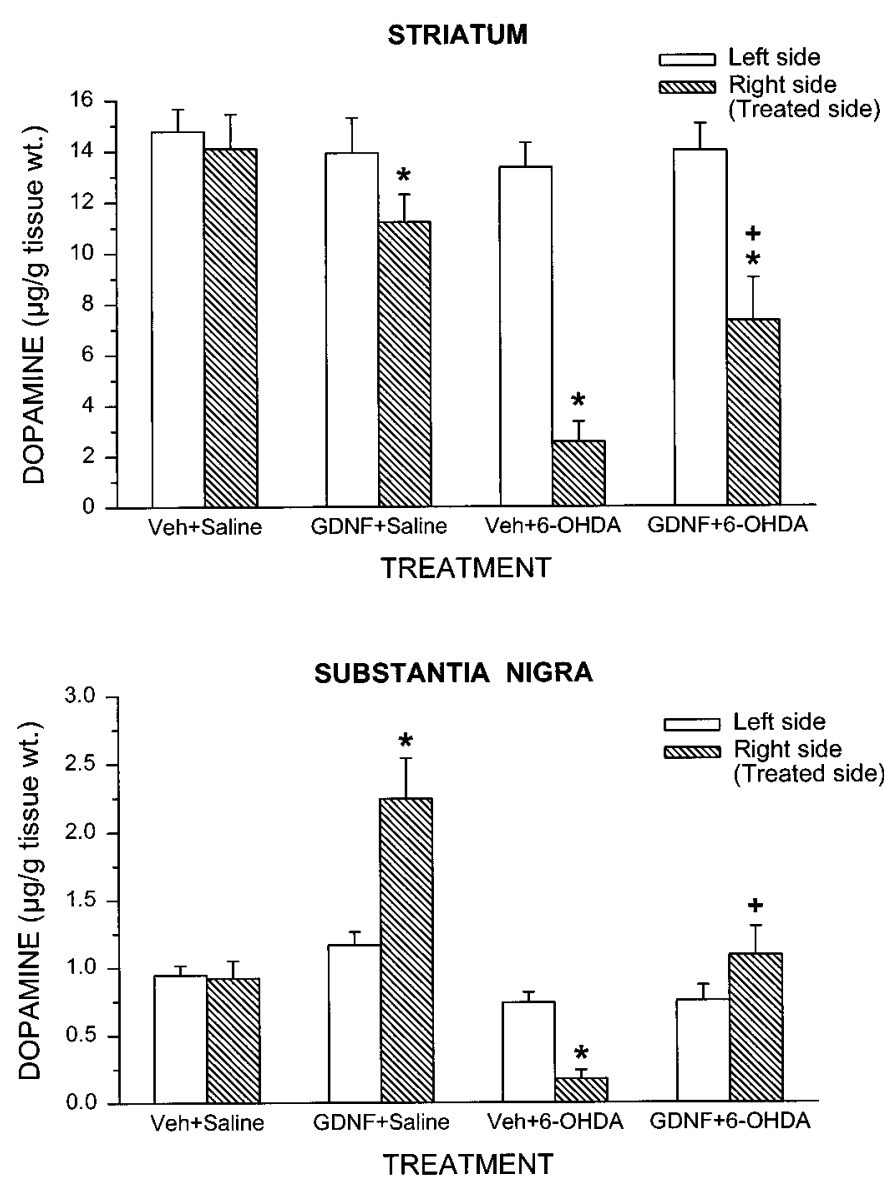

Figure 5. DA levels in the striatum (top) and substantia nigra (bottom) from rats treated 3-4 weeks earlier with intranigral GDNF or vehicle $(V e h)$ followed $6 \mathrm{hr}$ later by intranigral 6-OHDA or saline. All injections were made into the right side of the brain. The data shown are mean $( \pm$ SEM) values from eight animals per group. ${ }^{*} p<0.05$ versus the left side of the same group; $+p<0.05$ versus the right side of the vehicle + 6-OHDA group (two-factor ANOVA with side of the brain as a within factor; Newman-Keuls post hoc comparisons).

between the two sides of the brain at any recording depth (Fig. 6). In contrast, in animals treated with GDNF + 6-OHDA 10-12 weeks earlier, there was a significant decrease in signal amplitude in the treated dorsal striatum compared with the contralateral side (Fig. 6). Overall signal amplitudes and clearance rates from the striatum of the rats treated 10-12 weeks earlier are shown in Figure 7. There was no difference in average amplitude or clearance rate between the right and left striata in the GDNF + saline-treated animals. However, in the GDNF + 6-OHDAtreated animals, there was a $70 \%$ decrease in signal amplitude and a $69 \%$ decrease in clearance rate on the treated side of the brain compared with the contralateral side. Signal amplitudes and clearance rates from the treated side of these animals were significantly lower than those from the treated side of the GDNF +6 -OHDA group of animals that were examined at 3-4 weeks after treatment (Fig. 4; $p<0.05$, unpaired $t$ tests).

On the right side of the animals treated 10-12 weeks earlier with GDNF + saline, DA levels were decreased by $43 \%$ in the striatum and increased by $101 \%$ in the substantia nigra (Fig. 8). On the right side of the GDNF + 6-OHDA group, DA levels were decreased by $75 \%$ in the striatum and increased by $72 \%$ in the nigra compared with the contralateral side.
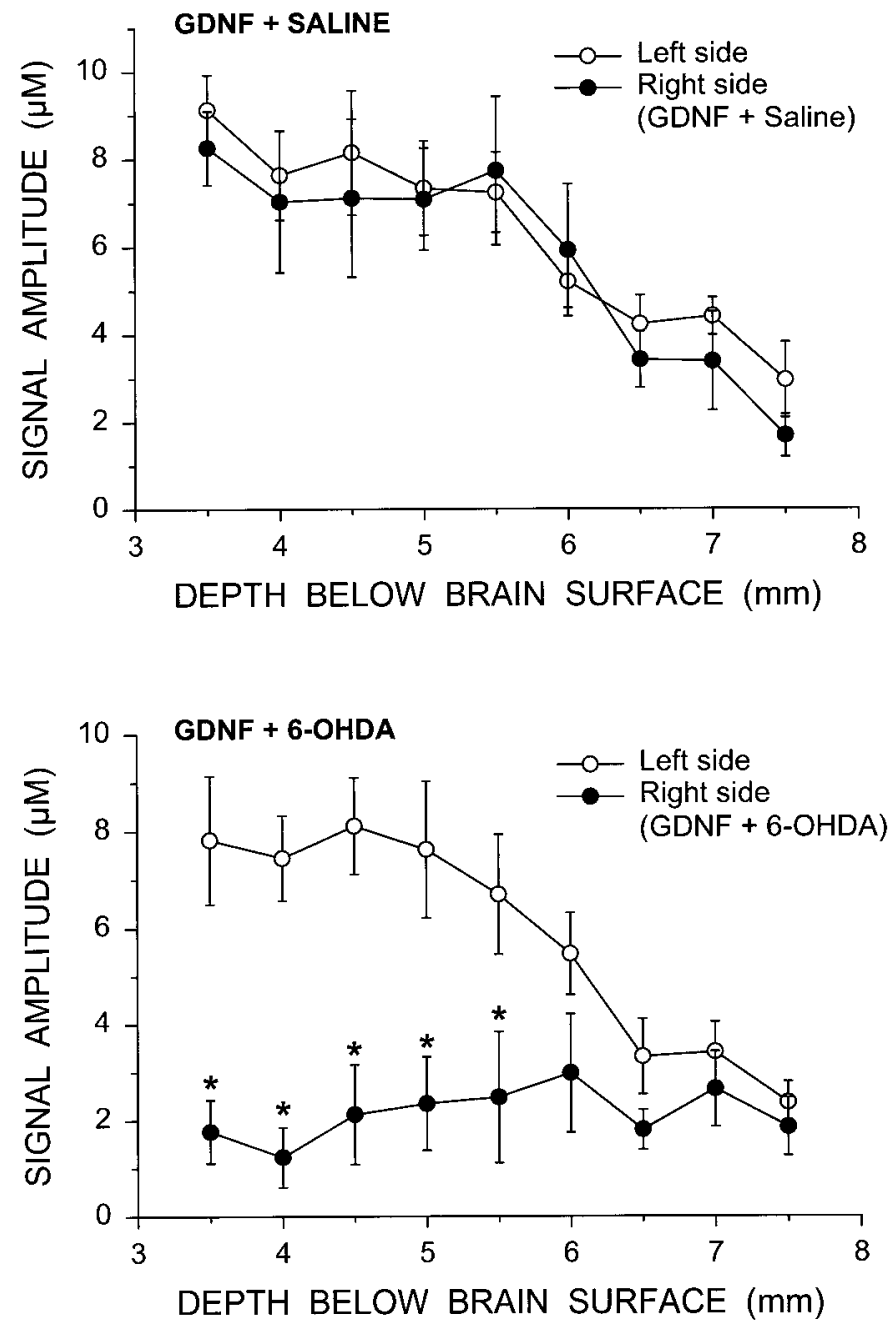

Figure 6. Summary of potassium-evoked DA signal amplitude throughout the striatum and nucleus accumbens of animals treated with GDNF followed $6 \mathrm{hr}$ later by saline (top) or 6-OHDA (bottom). All injections were made into the right substantia nigra. In vivo electrochemical recordings were made 10-12 weeks after the injections. The data shown are mean $( \pm$ SEM) values for eight animals per group. The data were analyzed using two-factor ANOVA with side of the brain and depth of the recording as within factors. $F$ scores for the $G D N F+$ saline group are as follows: side $F=0.29, p>0.05$; depth $F=7.01, p<0.001$; and interaction $F=0.60$, $p>0.05$. GDNF $+6-O H D A$ group $F$ scores are as follows: side $F=16.51$, $p<0.01$; depth $F=5.59, p<0.001$; and interaction $F=6.24, p<0.001$. ${ }^{*} p<0.05$ versus the left side at the same depth (Newman-Keuls post hoc comparisons).

\section{DISCUSSION}

GDNF is a potent neurotrophic factor that has significant effects on dopaminergic, as well as nondopaminergic, neurons in the mammalian CNS (Cass et al., 1998). The positive effects of GDNF on dopaminergic systems have led to the suggestion that GDNF may prove useful for the treatment of Parkinson's disease. It may be able to halt or slow down progression of the disease or even partially restore lost function. In the present study the protective effects of GDNF on the functioning of striatal DA terminals were evaluated in a rodent model of Parkinson's disease. Within the constraints of the paradigm used in the present study, our results indicate that although a single intranigral injection of GDNF may protect DA neuron cell bodies in the substan- 

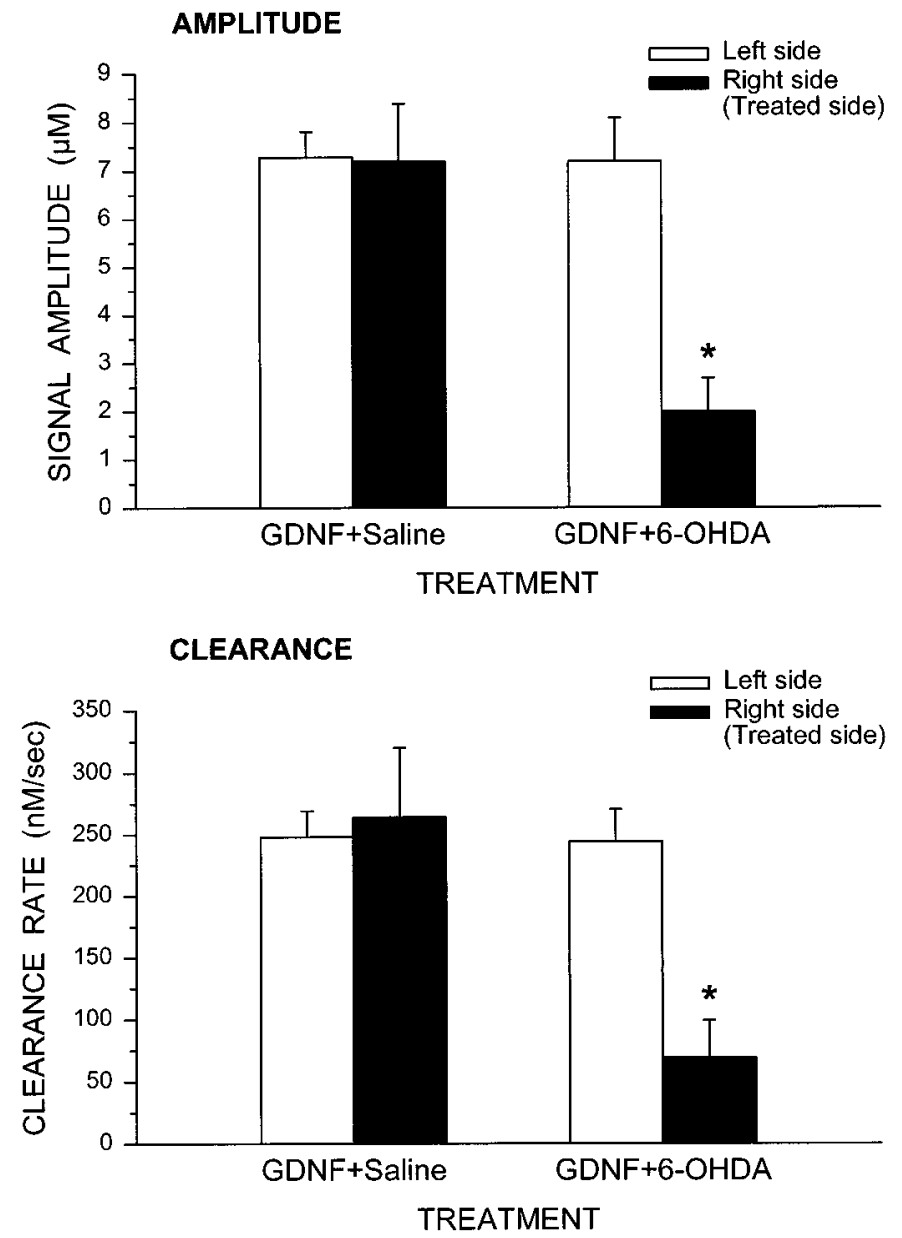

Figure 7. Signal amplitudes (top) and clearance rates (bottom) from the striatum of rats treated with $G D N F$ followed $6 \mathrm{hr}$ later by $6-O H D A$ or saline. All injections were made into the right substantia nigra. In vivo electrochemical recordings were made 10-12 weeks after the injections. The data from each recording depth in the striatum $(3.5-6.0 \mathrm{~mm}$ below the surface of the cortex) of each animal were averaged together. The values shown are mean $\left( \pm\right.$ SEM) for eight animals per group. ${ }^{*} p<0.05$ versus the left side of the same group (two-factor ANOVA with side of the brain as a within factor; Newman-Keuls post hoc comparisons).

tia nigra, the release of DA in the striatum is only minimally protected.

The time course for injection of GDNF, $6 \mathrm{hr}$ before 6-OHDA, was chosen on the basis of previous work that demonstrated that this time point preserved $\mathrm{TH}$-positive cell bodies in the nigra to a greater extent than did $0,1,12$, or $24 \mathrm{hr}$ time points (Kearns et al., 1997). In the present study, GDNF injected into the nigra $6 \mathrm{hr}$ before intranigral 6-OHDA prevented 6-OHDA-induced reductions in nigral DA levels at both 3-4 and 10-12 weeks after treatment. These results, along with the previous results indicating that this GDNF treatment completely prevents 6-OHDAinduced loss of DA cell number in the nigra (Kearns et al., 1997), suggest that a single injection of GDNF can provide complete protection to DA neuron cell bodies in the substantia nigra.

Other investigators have also shown that GDNF can provide near complete to complete protection of DA cell bodies in the nigra. Supranigral administration of GDNF every other day for 4 weeks, starting on the day of an intrastriatal 6-OHDA lesion, completely prevented loss of cells in the substantia nigra, whereas a single injection $7 \mathrm{~d}$ after the lesion partially prevented cell loss
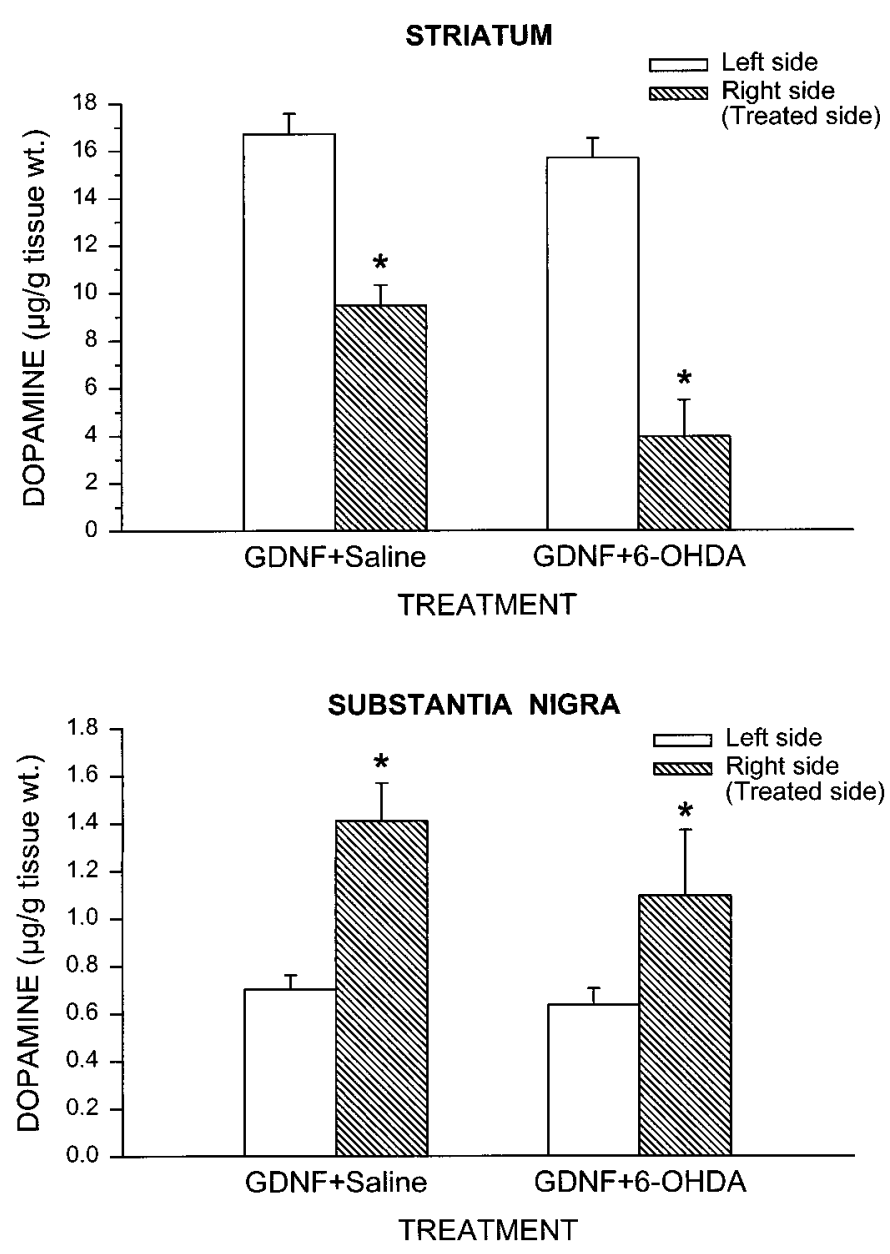

Figure 8. DA levels in the striatum (top) and substantia nigra (bottom) from rats treated 10-12 weeks earlier with intranigral $G D N F$ followed 6 hr later by intranigral 6-OHDA or saline. All injections were made into the right side of the brain. The data shown are mean ( \pm SEM) values from eight animals per group. ${ }^{*} p<0.05$ versus the left side of the same group (two-factor ANOVA with side of the brain as a within factor; Newman-Keuls post hoc comparisons).

(Sauer et al., 1995). When GDNF was injected into the striatum both before and after the intrastriatal administration of 6-OHDA, the number of TH-positive neurons in the substantia nigra was almost completely preserved (Shults et al., 1996). Multiple supranigral injections of GDNF for $37 \mathrm{~d}$ after intrastriatal 6-OHDA led to a 78\% sparing of nigral TH-positive neurons at 5 months after the lesion (Winkler et al., 1996). However, in these same animals there was no protective effect of GDNF on striatal DA terminals (Winkler et al., 1996). Ipsilateral administration of GDNF supranigrally and intraventricularly immediately before injection of 6-OHDA into the medial forebrain bundle led to a $77-80 \%$ survival of $\mathrm{TH}$-positive neurons in the midbrain and a $77 \%$ sparing of DA levels in the striatum 13 weeks after the lesion (Sullivan et al., 1998). These studies, like the present one, all support that GDNF can substantially protect nigral DA neurons from the neurotoxic effects of 6-OHDA.

Although the present GDNF treatment protected DA cell bodies, the results of the in vivo electrochemical studies suggest that presynaptic dopaminergic function is only partially protected. In the GDNF + 6-OHDA-treated rats, potassium-evoked overflow of DA and clearance of DA in the striatum decreased 
from 3-4 to 10-12 weeks after treatment. By 10-12 weeks after treatment, these indices of DA terminal function were not significantly different from those in the vehicle + 6-OHDA-treated animals at 3-4 weeks after the lesion (unpaired $t$ tests). Striatal DA levels declined in a similar manner over time. The decrease in dopaminergic functioning in the striatum over time suggests that some of the protective effects of GDNF in the striatum may actually represent an upregulation in the functioning of remaining DA terminals that declines over time. It has been shown recently that GDNF can increase the quantal size for release of DA from cultured midbrain neurons (Pothos et al., 1998). If this is a transient phenomenon that lasts only a few weeks, then this could explain the present results. Another possibility is that GDNF may initially provide protection to some striatal terminals but that over time the protective ability of GDNF diminishes while terminals may still be degenerating. Thus, although DA cell bodies in the nigra appear to be fully protected, DA terminals in the striatum are only partially protected. Further experiments will be necessary to determine the mechanisms responsible for the decrease in GDNF protection after 10-12 weeks.

The site of GDNF administration appears to be important for determining the effectiveness of GDNF in protecting striatal DA terminals. In the present study, intranigral GDNF provided minimal long-term protection to DA terminals. Sauer et al. (1995) and Winkler et al. (1996) reported similar results after supranigral GDNF. On the other hand, the combined injection of GDNF both above the nigra and into the lateral ventricle immediately before injection of 6-OHDA into the medial forebrain bundle led to a substantial sparing of DA levels in the striatum both at 2 weeks (Opacka-Juffry et al., 1995) and 13 weeks (Sullivan et al., 1998) after lesioning. In vivo indices of DA terminal functioning (positron emission tomography scans with a DA transporter tracer and striatal microdialysis) also indicated that the combined supranigral and intraventricular injection of GDNF provided significant protection to striatal DA terminals (Opacka-Juffry et al., 1995; Sullivan et al., 1998). In addition, the intrastriatal injection of an adenoviral vector expressing GDNF provided substantial sparing of $\mathrm{TH}$ immunoreactivity in the striatum against the effects of intrastriatal 6-OHDA (Bilang-Bleuel et al., 1997). Injection of a similar vector into the midbrain, while reducing 6-OHDA-induced loss of DA neurons in the nigra, did not appear to spare striatal TH immunoreactivity (ChoiLundberg et al., 1997; Mandel et al., 1997). Taken together with the present results, the evidence to date suggests that GDNF needs to be administered into the striatum, or adjacent to it, to provide significant protection to DA terminals against the effects of 6-OHDA.

In the GDNF + saline-treated animals, release and clearance of DA were not significantly altered on the GDNF-treated side compared with the contralateral side at either $3-4$ or $10-12$ weeks after treatment. In addition, there was no difference in release or clearance between the GDNF + saline-treated animals and the vehicle + saline-treated animals. Thus, in contrast to other reports (Hebert et al., 1996; Hebert and Gerhardt, 1997), we did not find a significant increase in evoked DA overflow in normal animals given GDNF. However, we did find that a single, intranigral injection of GDNF in normal animals produces long-term changes in tissue levels of DA. There was a significant decrease in striatal DA content at both 3-4 and 10-12 weeks after treatment but normal levels of potassium-evoked release of DA. This may indicate that DA synthesis is decreased in the striatum or that less DA is being stored in nonreleasable pools. Other investigators have also found a decrease, or a trend for a decrease, in striatal DA levels after GDNF administration (Hudson et al., 1995; Hebert et al., 1996; Martin et al., 1996).

The present result of an increase in nigral DA levels after intranigral GDNF treatment in nonlesioned animals is similar to that reported by other investigators (Gash et al., 1995; Hudson et al., 1995; Martin et al., 1996; Hebert and Gerhardt, 1997). The increase in DA content in the nigra, along with increased $\mathrm{TH}$ immunoreactivity in the nigra (Gash et al., 1995; Hudson et al., 1995), suggests that DA synthesis and storage may be increased in the nigra. One possible explanation for these results is that GDNF has increased DA synthesis in both the soma and terminal regions of DA neurons. In the cell bodies this leads to an accumulation of DA, whereas in the terminals there is an increase in DA turnover (Hudson et al., 1995) that leads to decreases in the stored levels of DA but to normal levels of extracellular DA. Another possible explanation for these results is that axoplasmic transport of TH to the terminals is diminished. This could lead to a buildup of TH and DA levels in the nigra and to a decrease in DA levels in the striatum. Presynaptic compensatory mechanisms may be maintaining normal extracellular levels of DA in the striatum (Zigmond et al., 1990). Further studies are needed to determine the nature and physiological significance of GDNFinduced changes in DA levels in the nigra and striatum of normal animals.

The present results suggest that a single intranigral administration of GDNF can prevent 6-OHDA-induced damage to nigral DA cell bodies. However, damage to striatal DA terminals is still present, and its extent may increase over time. It is possible that GDNF needs to be administered into the striatum, or adjacent to it in the ventricles, to afford substantial protection to DA terminals. These data further support that survival of DA cell bodies after neurotoxin lesioning does not necessarily indicate that DA terminals are functioning in their normal capacity.

\section{REFERENCES}

Beck KD, Valverde J, Alexi T, Poulsen K, Moffat B, Vandlen RA, Rosenthal A, Hefti F (1995) Mesencephalic dopaminergic neurons protected by GDNF from axotomy-induced degeneration in the adult brain. Nature 373:339-341.

Bilang-Bleuel A, Revah F, Colin P, Locquet I, Robert J-J, Mallet J, Horellou P (1997) Intrastriatal injection of an adenoviral vector expressing glial-cell-line-derived neurotrophic factor prevents dopaminergic neuron degeneration and behavioral impairment in a rat model of Parkinson's disease. Proc Natl Acad Sci USA 94:8818-8823.

Bowenkamp KE, Hoffman AF, Gerhardt GA, Henry MA, Biddle P, Hoffer BJ, Granholm A-CE (1995) Glial cell line-derived neurotrophic factor supports survival of injured midbrain dopaminergic neurons. J Comp Neurol 355:479-489.

Cass WA (1996) GDNF selectively protects dopamine neurons over serotonin neurons against the neurotoxic effects of methamphetamine. J Neurosci 16:8132-8139.

Cass WA (1997) Decreases in evoked overflow of dopamine in rat striatum after neurotoxic doses of methamphetamine. J Pharmacol Exp Ther 280:105-113.

Cass WA, Gerhardt GA (1994) Direct in vivo evidence that D2 dopamine receptors can modulate dopamine uptake. Neurosci Lett 176:259-263.

Cass WA, Gerhardt GA (1995) In vivo assessment of dopamine uptake in rat medial prefrontal cortex: comparison with dorsal striatum and nucleus accumbens. J Neurochem 65:201-207.

Cass WA, Gerhardt GA, Mayfield RD, Curella P, Zahniser NR (1992) Differences in dopamine clearance and diffusion in rat striatum and nucleus accumbens following systemic cocaine administration. J Neurochem 59:259-266.

Cass WA, Zahniser NR, Flach KA, Gerhardt GA (1993) Clearance of exogenous dopamine in rat dorsal striatum and nucleus accumbens: 
role of metabolism and effects of locally applied uptake inhibitors. J Neurochem 61:2269-2278.

Cass WA, Kearns CM, Gash DM (1998) Protective and regenerative properties of GDNF in the central nervous system. In: Neuroprotective signal transduction (Mattson MP, ed), pp 145-161. Totowa, NJ: Humana.

Choi-Lundberg DL, Lin Q, Chang Y-N, Chiang YL, Hay CM, Mohajeri H, Davidson BL, Bohn MC (1997) Dopaminergic neurons protected from degeneration by GDNF gene therapy. Science 275:838-841.

Friedemann MN, Gerhardt GA (1992) Regional effects of aging on dopaminergic function in the Fischer-344 rat. Neurobiol Aging 13:325-332.

Garris PA, Walker QD, Wightman RM (1997) Dopamine release and uptake rates both decrease in the partially denervated striatum in proportion to the loss of dopamine terminals. Brain Res 753:225-234.

Gash DM, Zhang Z, Cass WA, Ovadia A, Simmerman L, Martin D, Russell D, Collins F, Hoffer BJ, Gerhardt GA (1995) Morphological and functional effects of intranigrally administered GDNF in normal rhesus monkeys. J Comp Neurol 363:345-358.

Gash DM, Zhang Z, Ovadia A, Cass WA, Yi A, Simmerman L, Russell D, Martin D, Lapchak PA, Collins F, Hoffer BJ, Gerhardt GA (1996) Functional recovery in GDNF-treated parkinsonian monkeys. Nature 380:252-255.

Gerhardt GA, Oke AF, Nagy G, Moghaddam B, Adams RN (1984) Nafion-coated electrodes with high selectivity for CNS electrochemistry. Brain Res 290:390-395.

Hebert MA, Gerhardt GA (1997) Behavioral and neurochemical effects of intranigral administration of glial cell line-derived neurotrophic factor on aged Fischer 344 rats. J Pharmacol Exp Ther 282:760-768.

Hebert MA, Van Horne CG, Hoffer BJ, Gerhardt GA (1996) Functional effects of GDNF in normal rat striatum: presynaptic studies using in vivo electrochemistry and microdialysis. J Pharmacol Exp Ther 279:1181-1190.

Hoffer BJ, Hoffman A, Bowenkamp K, Huettl P, Hudson J, Martin D, Lin L-FH, Gerhardt GA (1994) Glial cell line-derived neurotrophic factor reverses toxin-induced injury to midbrain dopaminergic neurons in vivo. Neurosci Lett 182:107-111.

Hou J-GG, Lin L-FH, Mytilineou C (1996) Glial cell line-derived neurotrophic factor exerts neurotrophic effects on dopaminergic neurons in vitro and promotes their survival and regrowth after damage by 1-methyl-4-phenylpyridinium. J Neurochem 66:74-82.

Hudson J, Granholm A-C, Gerhardt G, Henry MA, Hoffman A, Biddle P, Leela NS, Mackerlova L, Lile JD, Collins F, Hoffer BJ (1995) Glial cell line-derived neurotrophic factor augments midbrain dopaminergic circuits in vivo. Brain Res Bull 36:425-432.

Kearns CM, Gash DM (1995) GDNF protects nigral dopamine neurons against 6-hydroxydopamine in vivo. Brain Res 672:104-111.

Kearns CM, Cass WA, Smoot K, Kryscio R, Gash DM (1997) GDNF protection against 6-OHDA: time dependence and requirement for protein synthesis. J Neurosci 17:7111-7118.

Kojima H, Abiru Y, Sakajiri K, Watabe K, Ohishi N, Takamori M, Hatanaka H, Yagi K (1997) Adenovirus-mediated transduction with human glial cell line-derived neurotrophic factor gene prevents 1-methyl-4-phenyl-1,2,3,6-tetrahydropyridine-induced dopamine depletion in striatum of mouse brain. Biochem Biophys Res Commun 238:569-573.

Lapchak PA, Miller PJ, Collins F, Jiao S (1997) Glial cell line-derived neurotrophic factor attenuates behavioral deficits and regulates nigrostriatal dopaminergic and peptidergic markers in 6-hydroxydopaminelesioned adult rats: comparison of intraventricular and intranigral delivery. Neuroscience 78:61-72.
Lin L-FH, Doherty DH, Lile JD, Bektesh S, Collins F (1993) GDNF: a glial cell line-derived neurotrophic factor for midbrain dopaminergic neurons. Science 260:1130-1132.

Luthman J, Friedemann M, Bickford P, Olson L, Hoffer BJ, Gerhardt GA (1993) In vivo electrochemical measurements and electrophysiological studies of rat striatum following neonatal 6-hydroxydopamine treatment. Neuroscience 52:677-687.

Mandel RJ, Spratt SK, Snyder RO, Leff SE (1997) Midbrain injection of recombinant adeno-associated virus encoding rat glial cell line-derived neurotrophic factor protects nigral neurons in a progressive 6-hydroxydopamine-induced degeneration model of Parkinson's disease in rats. Proc Natl Acad Sci USA 94:14083-14088.

Martin D, Miller G, Cullen T, Fischer N, Dix D, Russell D (1996) Intranigral or intrastriatal injections of GDNF: effects on monoamine levels and behavior in rats. Eur J Pharmacol 317:247-256.

Miyoshi Y, Zhang Z, Ovadia A, Lapchak PA, Collins F, Hilt D, Lebel C, Kryscio R, Gash DM (1997) Glial cell line-derived neurotrophic factor-levodopa interactions and reduction of side effects in parkinsonian monkeys. Ann Neurol 42:208-214.

Opacka-Juffry J, Ashworth S, Hume SP, Martin D, Brooks DJ, Blunt SB (1995) GDNF protects against 6-OHDA nigrostriatal lesion: in vivo study with microdialysis and PET. NeuroReport 7:348-352.

Pothos NP, Davila V, Sulzer D (1998) Presynaptic recording of quanta from midbrain dopamine neurons and modulation of quantal size. J Neurosci 18:4106-4118.

Rosenblad C, Martinez-Serrano A, Björklund A (1998) Intrastriatal glial cell line-derived neurotrophic factor promotes sprouting of spared nigrostriatal dopaminergic afferents and induces recovery of function in a rat model of Parkinson's disease. Neuroscience 82:129-137.

Sauer H, Rosenblad C, Bjorklund A (1995) Glial cell line-derived neurotrophic factor but not transforming growth factor $\beta 3$ prevents delayed degeneration of nigral dopaminergic neurons following striatal 6-hydroxydopamine lesions. Proc Natl Acad Sci USA 92:8935-8939.

Shults CW, Kimber T, Martin D (1996) Intrastriatal injection of GDNF attenuates the effects of 6-hydroxydopamine. NeuroReport 7:627-631.

Suaud-Chagny MF, Dugast C, Chergui K, Msghina M, Ganon F (1995) Uptake of dopamine released by impulse flow in the rat mesolimbic and striatal systems in vivo. J Neurochem 65:2603-2611.

Sullivan AM, Opacka-Juffry J, Blunt SB (1998) Long-term protection of the rat nigrostriatal dopaminergic system by glial cell line-derived neurotrophic factor against 6-hydroxydopamine in vivo. Eur J Neurosci 10:57-63.

Tomac A, Lindqvist E, Lin L-FH, Ogren SO, Young D, Hoffer BJ, Olson L (1995) Protection and repair of the nigrostriatal dopaminergic system by GDNF in vivo. Nature 373:335-339.

Wightman RM, Zimmerman JB (1990) Control of dopamine extracellular concentration in rat striatum by impulse flow and uptake. Brain Res Rev 15:135-144.

Winkler C, Sauer H, Lee CS, Björklund A (1996) Short-term GDNF treatment provides long-term rescue of lesioned nigral dopaminergic neurons in a rat model of Parkinson's disease. J Neurosci 16:7206-7215.

Zhang Z, Miyoshi Y, Lapchak PA, Collins F, Hilt D, Lebel C, Kryscio R, Gash DM (1997) Dose response to intraventricular glial cell linederived neurotrophic factor administration in parkinsonian monkeys. J Pharmacol Exp Ther 282:1396-1401.

Zigmond MJ, Abercrombie ED, Berger TW, Grace AA, Stricker EM (1990) Compensations after lesions of central dopaminergic neurons: some clinical and basic implications. Trends Neurosci 13:290-296. 